\title{
Diversidad de uso tecnológico en el alumnado universitario; lo académico vs. lo personal
}

\author{
Diversity of technological use in university students; \\ the academic vs. the personal
}

Ainara Romero Andonegui

ainara.romero@ehu.eus

Naiara Bilbao Quintana

naiara.bilbao@ehu.eus

(D) Eneko Tejada Garitano

eneko.tejada@ehu.eus

Arantzazu López de la Serna

arantzazu.lopez@ehu.eus

Departamento de Didáctica y Organización Escolar, Universidad del País Vasco, España

\section{RESUMEN}

La integración de la tecnología en el ámbito universitario requiere tanto de herramientas tecnológicas como de diseño pedagógico que lleve a prácticas efectivas. Nuestras universidades han invertido en recursos y en formación para ello, pero la repercusión esperada en los procesos de enseñanza-aprendizaje está tardando en darse. En este estudio se quiere partir de la percepción del alumnado sobre los recursos relacionados con las Tecnologías de la Información y la Comunicación (TIC) universitarios y su uso, y comparar las expectativas y uso que realizan en este entorno académico con el que realizan en su ámbito personal. Participan en el estudio 186 estudiantes del Grado de Educación a los que se les facilitó el test ECAR. Los resultados muestran que el alumnado desconoce gran parte de los recursos TIC universitarios y las posibilidades pedagógicas que éstos ofrecen. Se subraya que los estudiantes prefieren una enseñanza presencial y con una utilización mínima de tecnología, a pesar de reconocer su implicación en el éxito académico. También se ha encontrado que las expectativas y uso que realiza de los recursos TIC en el plano académico difiere sustancialmente al que hace fuera del aula. En líneas futuras, habría que resolver por qué el alumnado desconoce los recursos TIC universitarios y sus posibilidades educativas y en qué medida afecta el uso e integración de la tecnología que hace el profesorado en las expectativas y uso de ellas por parte del alumnado.

PALABRAS CLAVE tecnología, educación superior, uso, contexto.

ABSTRACT

The integration of technology in the university environment requires both technological tools and pedagogical design that leads to effective practices. Our universities have invested in resources and training for this, but the expected repercussion 
in the teaching-learning processes is taking time. In this study we want to start from the perception of the students about the university ICT resources and their use, and compare the expectations and use they make in this academic environment with the one they perform in their personal field. 186 students of the Degree in Education participated in the study and were given the ECAR test. The results of the descriptive and correlational analysis show that students ignore part of the university ICT resources and the pedagogical possibilities they offer. They prefer face-to-face teaching with minimal use of technology. It also found that the expectations and use made of ICT resources at the academic level differ substantially from what it does outside the classroom. In future lines, it would be necessary to solve why students do not know the university ICT resources and their educational possibilities and to what extent it affects the use and integration of the technology made by teachers in the expectations and use of them by students.

KEYWORDS technology, higher education, use, context.

\section{INTRODUCCIÓN}

Milennials (Howe, \& Strauss, 1991), Generación Net (Tapscott, 1998) o Nativos Digitales (Prensky, 2001) son algunos de los términos que han acuñado diferentes autores para referirse a las personas nacidas en la década de los ochenta y noventa.

Toda esta terminología fundamentalmente hace referencia a la primera generación que ha crecido rodeada de tecnología y que, de forma particular, muestra familiaridad y confianza hacia las Tecnologías de la Información y Comunicación (TIC) (Gallardo et al., 2016). Sin embargo, este hecho no predispone a estos jóvenes a poseer una competencia digital innata, es decir, "la generación sola no define adecuadamente si alguien es un nativo digital o no" (Helsper, \& Eynon, 2010, p.515), ya que el uso de la tecnología, por sí mismo, por muy natural e intensivo que sea, no capacita en la práctica correcta de las TIC.

Por lo tanto, deben quedar atrás las atribuciones realizadas en torno a toda esa nomenclatura y a la idea de que los actuales estudiantes universitarios han desarrollado capacidades inherentes para el uso de las TIC en todos los contextos de su vida, sólo por el mero hecho de haber nacido rodeados de tecnología (Gallardo et al., 2015).

\subsection{El estudiante universitario en la era digital}

Hoy sabemos que el patrón de acceso y uso de la tecnología por parte de los estudiantes es diverso. Esto se traduce en que un mismo estudiante utiliza la tecnología de forma diferente en función del contexto (Romero et al., 2017). No obstante, en la literatura científica sobre el uso y actitud que tiene el alumnado universitario respecto a las TIC, se encuentran investigaciones con posturas y resultados contrapuestos. De esta forma, algunos estudios sugieren que los estudiantes universitarios poseen una cultura propia de participación, destrezas para acceder de forma rápida a la información, capacidades para adherirse a nuevas vías comunicativas que responden a sus necesidades, y un perfil de carácter prosumer, es decir, orientado a la producción de contenido (Erstad, 2010; Pedró, 2009). Por contra, otros trabajos señalan que, a pesar de que los estudiantes tienen un mayor acceso a las fuentes de información, no saben gestionarla e interpretarla de forma adecuada y que sólo una minoría de los universitarios es partidaria de crear contenido en la red (Bennet et al., 2008; Boyd, 2014). 
En cualquiera de los casos, las diferentes investigaciones sí recogen que los estudiantes tienen una actitud y usan las TIC en contexto personal de forma diferente al que lo hacen en el ámbito de trabajo o aprendizaje formal (Corrin, 2014; Kennedy et al., 2007; Waycott et al., 2010). Se entiende que el alumnado no transfiere sus habilidades y destrezas al mundo académico en términos de aprendizaje (Gisbert, \& Esteve, 2016; Waycott et al., 2010) y que las actividades digitales que realiza reflejan múltiples dimensiones en uno u otro sentido (Hietajärvi et al., 2019).

Por todo ello, los cambios en la enseñanza universitaria deben comprenderse a partir de cómo traducen los estudiantes el uso de las tecnologías en el ámbito personal al del contexto de aprendizaje formal (Kumar, 2009).

\subsection{Los desafíos en Educación Superior con la tecnología educativa}

La Tecnología de la Información y Comunicación se ha insertado en todas las esferas de la Educación Superior. Y es que además de haber influido de forma notable en la administración y gestión universitaria, también lo ha hecho en el ámbito pedagógico.

En este último sentido, cabe destacar que en la actualidad el alumnado tiene a su alcance diferentes modalidades de formación y que hoy, más que nunca, dispone de medios de calidad que contribuyen a su instrucción formal (Tejada, \& Maíz, 2013). Sin embargo, para hacer un uso adecuado de todos estos recursos tecnológicos y beneficiarse de todas sus potencialidades, todavía hoy en día, se necesita de un cambio de paradigma en la enseñanza superior, tanto en el profesorado como en los estudiantes (Sánchez et al., 2013).

Si bien las universidades han destinado partidas presupuestarias a implantar Campus Virtuales y fomentar la creación de Espacios Virtuales de Aprendizaje (EVA) como Open Course Wares (OCW) y Massive Open Online Courses (MOOC), etc., el cambio en el uso, actitud y sobre todo la implementación de las TIC a nivel metodológico por parte del profesorado, está tardando en darse. Y es que todavía son muchas las plataformas universitarias que se utilizan más como repositorios que como entornos de enseñanza-aprendizaje (Vázquez et al., 2016).

El mero uso de la tecnología, per se, no contribuye a que esta se integre pedagógicamente. Como afirman Gros y Noguera (2015), la elección de tecnología educativa emergente en las propuestas de enseñanzaaprendizaje debe fundamentarse en la implementación de metodologías activas, es decir, en prácticas de enseñanza-aprendizaje efectivas que pongan el acento en el estudiante y en las acciones que realiza por medio de ellas con objeto de formarse (Hernández, \& Torrijas, 2018).

Por su parte, los estudiantes también tienen desafíos, ya que deben implicarse activamente y asumir la cuota de responsabilidad que les corresponde como sujetos activos en proceso de formación. Es por ello, que el Informe Horizon (2018) sigue señalando como objetivo el desarrollo de la competencia digital del estudiante universitario, ya que éstos presentan dificultad para instrumentalizar sus habilidades digitales en su proceso de aprendizaje y, en general, en el desarrollo de su formación (Gisbert, \& Esteve, 2016). 
Por todo ello, es necesario conocer si los esfuerzos de la universidad y del profesorado universitario para integrar la tecnología en el proceso de enseñanza-aprendizaje influyen en el uso y actitud del alumnado hacia las TIC en este contexto. Así pues, en este estudio se pretende dar respuesta a dos cuestiones: a) ¿Qué opina el alumnado de los recursos TIC que ofrece la universidad?, b) ¿El alumnado universitario es capaz de trasladar el uso y expectativas en torno a la tecnología desde el ámbito personal al académico?

\section{OBJETIVOS E HIPÓTESIS}

La siguiente investigación persigue identificar y comparar la percepción sobre el uso y expectativas de la tecnología en el ámbito personal y en el académico por parte de los estudiantes universitarios.

Para ello se han planteado las siguientes hipótesis;

- H1: Los estudiantes se muestran satisfechos con los recursos tecnológicos que implementa el profesorado en el proceso de enseñanza-aprendizaje.

- H2: Los estudiantes consideran beneficioso el uso de la tecnología para el desarrollo de su formación académica y futuro profesional.

- H3: El uso de las tecnologías en el ámbito personal es diferente al ámbito académico.

\section{METODOLOGÍA}

Se plantea un estudio de carácter descriptivo para identificar la disponibilidad por parte del alumnado de herramientas y recursos necesarios para utilizar la tecnología en todos los ámbitos (social y académico) y, en concreto, aquellos disponibles en la universidad. Por otro lado, también se realiza un estudio correlacional para analizar la relación entre las expectativas y uso de la tecnología que realizan en el entorno académico con el que realizan en su ámbito personal.

\subsection{Muestra}

La muestra no probabilística de corte incidental la componen 186 alumnos y alumnas de la Facultad de Educación de Bilbao (UPV/EHU; España; Vizcaya) que cursan los grados de Educación Infantil y Educación Primaria. Un 66.44\% son mujeres y casi el 70\% se sitúa entre los 18 y 20 años (Tabla 1 ).

TABLA 1. Distribución de la muestra

\begin{tabular}{cccccc}
\hline Muestra & Educación & Educación & \multicolumn{3}{c}{ Edad } \\
\cline { 4 - 6 } & Primaria & Infantil & $18-20$ & $20-25$ & $25-30$ \\
\hline Mujeres & 75 & 43 & 89 & 29 & 0 \\
\hline Hombres & 18 & 50 & 40 & 24 & 4 \\
\hline Total & 93 & 93 & 129 & 53 & 4 \\
\hline
\end{tabular}

Los criterios de inclusión fueron haber cursado la asignatura de "Nuevas Tecnologías Aplicadas a la Educación" que se da en segundo de carrera. Con ello, se pretendía que todos los participantes tuvieran al menos un año y medio de experiencia universitaria y que hubiesen tenido opción de acceder a los diferentes recursos TIC que dispone en la universidad. 


\subsection{Instrumentos}

Para la investigación se construyó un instrumento de recogida de información, elaborado a partir de la prueba ECAR, Study of Undergraduate Students and Technology (Brooks, \& Pomerantz, 2017). El cuestionario ECAR ha sido utilizado desde 2004 por el gobierno de los Estados Unidos para analizar la infraestructura TIC necesaria, desde el punto de vista de los estudiantes. Este instrumento busca comprender las preferencias y experiencias de los estudiantes.

A través del cuestionario se persigue realizar una exploración en la Educación Superior sobre el compromiso de los estudiantes con la tecnología. La encuesta consta de 38 preguntas divididas en cuatro secciones: a) la importancia de la tecnología; b) las experiencias con la tecnología universitaria; c) las preferencias y experiencias de los estudiantes con la tecnología educativa; d) preguntas sociodemográficas.

Para la adaptación, estandarización y traducción de la prueba a nuestro contexto lingüístico, el euskera (idioma cooficial junto al español de la Comunidad Autónoma Vasca), así como a los recursos disponibles y utilizados en nuestra facultad, se han tenido en cuenta las directrices de la Comisión Internacional de Tests (ITC). Asimismo, para evaluar la validez de la prueba, se han seguido las indicaciones de Escobar y Cuervo (2008), seleccionando el juicio de expertos como procedimiento para estimar la validez de contenido.

En total han participado 5 jueces, todos ellos expertos en tecnología educativa y con el perfil lingüístico superior de euskera (C2). Con el fin de facilitar su trabajo y recoger sus respuestas, se diseñó una rúbrica mediante el software Corubric (herramienta online que permite crear rúbricas), la cual medía la suficiencia, claridad, coherencia y relevancia de los ítems para cada dimensión, en base a unos rangos que iban de 1 (Poco) a 4 (mucho). A los jueces también se les facilitó la información relacionada con el uso que se iba a dar a la prueba.

Para medir el grado de asociación de las respuestas dadas en Corubric por los diferentes jueces, se analizó el coeficiente de concordancia W de Kendall. Las respuestas a las 38 preguntas planteadas tienen un nivel de concordancia superior a 0.7 , siendo la media 0.786 y $p<0.05$. Por lo que se concluye que hay concordancia entre los rangos asignados por los jueces, y se identifican de forma adecuada los indicadores de logro expuestos.

El Alfa de Cronbach es de 0.748 lo que muestra que la consistencia interna de los ítems es aceptable. Se recurrió igualmente al Alfa de Cronbach para cada dimensión identificando los ítems menos consistentes. Para la validez de constructo se sometió el instrumento a un análisis factorial exploratorio, aplicando el método máxima verosimilitud y rotación oblicua, con la intención de concentrar la pertenencia de los ítems a un factor y así discriminar mejor entre factores. Se eliminan 12 ítems con índice de saturación inferior a 0.40. Finalmente, la prueba ECAR adaptada consta de 26 preguntas distribuidas en cuatro factores/secciones, como se muestra en la tabla 2.

TABLA 2. Prueba ECAR adaptada

\begin{tabular}{ll}
\hline Sección & Preguntas \\
\hline $\begin{array}{l}\text { Propiedad, acceso y uso } \\
\text { del dispositivo }\end{array}$ & ¿Cuántos dispositivos dispone con acceso a Internet? \\
& ¿A cuántos de los siguientes dispositivos tiene acceso? \\
& De los dispositivos a los que tiene acceso, ¿cómo accede a ellos? \\
& En los últimos 12 meses, ¿qué uso le ha dado a cada dispositivo en su ámbito personal? \\
& En los últimos 12 meses, ¿qué uso le ha dado a cada dispositivo en su vida académica? \\
& ¿Cómo de importante es cada dispositivo para su éxito académico? \\
\hline
\end{tabular}




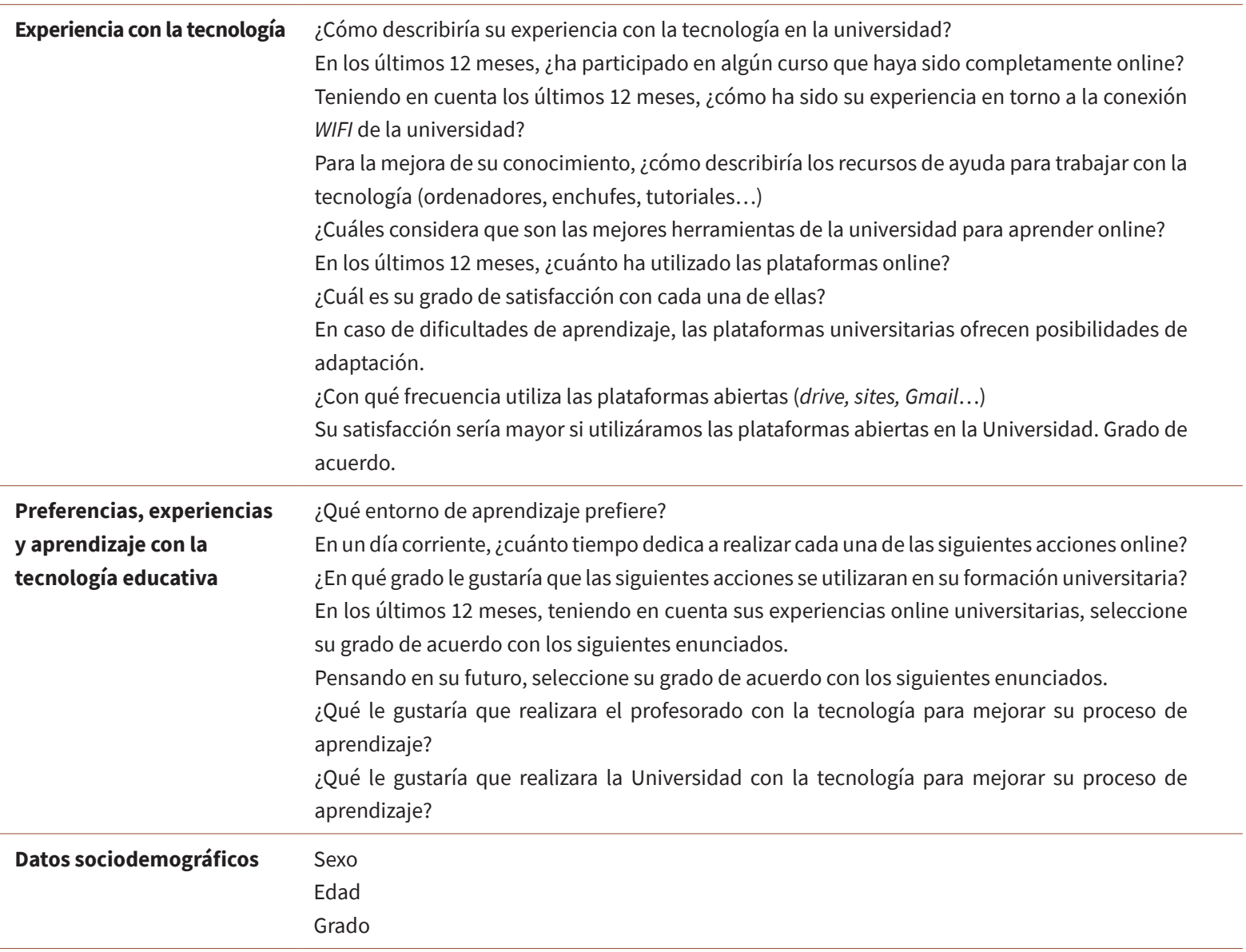

\subsection{Procedimiento de recolección de datos}

El cuestionario se digitalizó en la plataforma Google Form y en el mismo se adjuntaba el consentimiento informado para los participantes. El cuestionario se dispuso en la plataforma de la universidad, servicio que da soporte a las aulas virtuales de los estudios de Grado y Másteres Oficiales, además de en la página web de la asignatura "TIC para Educación”. Finalmente, de los 400 estudiantes que podían acceder a dichos entornos, un 46.5\% participó en el estudio, un total de 186 alumnos y alumnas. Los estudiantes tardaron una media de 16 minutos en cumplimentar los formularios. El análisis de los resultados se ha realizado mediante el software SPSS v.24.

\section{RESULTADOS}

\subsection{Descripción de la disponibilidad y uso de la tecnología}

La tabla 3 muestra las respuestas a los ítems 1, 2 y 3. Éstos indican que los encuestados tienen una media de entre 3 y 4 (3.7) dispositivos por persona y que la mayoría de los estudiantes disponen de un ordenador portátil y un teléfono Smartphone. Asimismo, la mitad de los encuestados disponen de ordenador de mesa y de tableta propios, y los restantes tienen a su disposición estos dispositivos por medio de 
TABLA 3. Alumnado que tiene acceso a cada dispositivo

\begin{tabular}{lcccc}
\hline & $\begin{array}{c}\text { Mi } \\
\text { propiedad }\end{array}$ & $\begin{array}{c}\text { Un } \\
\text { familiar }\end{array}$ & Universidad & $\begin{array}{c}\text { No } \\
\text { tengo }\end{array}$ \\
\hline $\begin{array}{l}\text { Ordenador } \\
\text { de mesa }\end{array}$ & 43.5 & 17.7 & 29.6 & 9.1 \\
\hline $\begin{array}{l}\text { Ordenador } \\
\text { portátil }\end{array}$ & 93 & 5.4 & 0 & 1.6 \\
\hline $\begin{array}{l}\text { Tableta } \\
\text { Smartphone }\end{array}$ & 100 & 0 & 0 & 0 \\
\hline Smartwatch & 10.2 & 5.9 & 0 & 83.9 \\
\hline $\begin{array}{l}\text { Disp. Real. } \\
\text { Virtual }\end{array}$ & 3.8 & 10.2 & 0 & 86 \\
\hline Disp. 3D & 8.1 & 2.2 & 0 & 89.8 \\
\hline Juegos & 32.8 & 18.3 & 0 & 48.9 \\
\hline $\begin{array}{l}\text { Disp. } \\
\text { Stream }\end{array}$ & 17.2 & 25.8 & 0 & 57 \\
\hline Disp. Voz & 3.8 & 6.5 & 0 & 89.8 \\
\hline
\end{tabular}

TABLA 4. Media y desviación típica de las respuestas sobre las experiencias con la tecnología de la Universidad

\begin{tabular}{lccccc}
\hline & N & Max & Min. & x & Desv. \\
\hline Exper_TecUniv & 186 & 1 & 6 & 3.34 & 1.013 \\
\hline Part_CursosOnline & 186 & 1 & 3 & 1.26 & .518 \\
\hline Exp_WIFI & 186 & 1 & 4 & 2.86 & 1.01 \\
\hline Exp_Rec & 186 & 1 & 5 & 3.30 & 1.170 \\
\hline Satis_eGela & 186 & 1 & 6 & 4.81 & .726 \\
\hline Satis_CampusVirtual & 186 & 1 & 6 & 1.09 & .407 \\
\hline Satis_GAUR & 186 & 1 & 6 & 4.55 & 1.003 \\
\hline Satis_OCW & 186 & 1 & 6 & 1.09 & .407 \\
\hline Satis_MiriadaX & 186 & 1 & 6 & 1.09 & .407 \\
\hline Valor_Adaptación & 186 & 1 & 6 & 3.67 & 1.421 \\
\hline Predis_RecAbierto & 186 & 1 & 5 & 3.96 & .866 \\
\hline
\end{tabular}
valorados de forma negativa por parte del alumnado; como son los MOOCs en Universidad-Miriadax ( $\mathrm{x}=1.09$ ), Open Course Ware ( $\mathrm{x}=1.09)$ o Campus Virtual $(\mathrm{x}=1.09)$. Habría que considerar si este resultado es fruto de su desconocimiento o insatisfacción con los esos recursos.

\subsection{El uso de las TIC en el desarrollo académico y profesional}

Respecto al uso de las TIC, los resultados indican que los estudiantes utilizan diariamente el ordenador $(x=4.34 ; \mathrm{s}=1.12)$ y el Smartphone $(\mathrm{x}=4.81, \mathrm{sd}=0.64)$ para actividades académicas. Del mismo modo, los encues- un familiar o de los recursos universitarios. Por el contrario, no disponen de Smartwatch, dispositivos 3D, controladores de voz o de realidad virtual.

Por lo tanto, los estudiantes disponen de las herramientas y recursos para utilizar la tecnología tanto en el ámbito académico como social.

\subsection{Valoración de la experiencia con tecnología en la Universidad}

Para el análisis del grado de satisfacción de los estudiantes respecto a los recursos tecnológicos (H1) se han analizado las respuestas dadas a las preguntas de la sección "Experiencias con la tecnología de la universidad" (tabla 4). El análisis realizado pone de manifiesto que los recursos tecnológicos que implementa el profesorado de la universidad en el proceso de enseñanza-aprendizaje son los que mejor valoración obtienen, ya que la plataforma corporativa eGela (Moodle) es la que mayor puntuación ha alcanzado $(x=4.81)$, siendo su valoración muy alta. Además, el alumnado también se muestra muy predispuesto a incorporar recursos y plataformas abiertas a su proceso de enseñanza-aprendizaje ( $x=3.96)$.

Por otra parte, la plataforma que utiliza el alumnado y profesorado para la gestión académica universitaria es valorada de forma muy satisfactoria $(x=4.55)$.

Así pues, aquellos recursos que conoce y utiliza de forma continua para su aprendizaje son valorados positivamente, aceptando la hipótesis 1 . Sin embargo, hay recursos tecnológicos que no se utilizan ordinariamente para una asignatura pero que están disponibles para el alumnado y que son 
TABLA 5. Media y desviación típica de las respuestas sobre las Preferencias, experiencias y aprendizaje con tecnología educativa

\begin{tabular}{|c|c|c|c|c|}
\hline & Mínimo & Máximo & Media & Desv. St. \\
\hline $\begin{array}{l}\text { Preferencia } \\
\text { Entorno }\end{array}$ & 1 & 6 & 1.85 & 1.254 \\
\hline $\begin{array}{l}\text { Preferencia } \\
\text { RRSS univ. }\end{array}$ & 1 & 5 & 2.63 & .990 \\
\hline $\begin{array}{l}\text { Preferencia } \\
\text { videos univ. }\end{array}$ & 1 & 4 & 2.51 & 1.077 \\
\hline $\begin{array}{l}\text { Preferencia } \\
\text { juegos univ. }\end{array}$ & 1 & 4 & 1.94 & .937 \\
\hline $\begin{array}{l}\text { Preferencia } \\
\text { activ. Colab. } \\
\text { Univ. }\end{array}$ & 1 & 5 & 3.19 & 1.078 \\
\hline $\begin{array}{l}\text { Preferencia } \\
\text { foros univ. }\end{array}$ & 1 & 4 & 2.72 & .992 \\
\hline
\end{tabular}

TABLA 6. Media y DT del uso de cada dispositivo en el ámbito personal y académico (escala del 1-5)

\begin{tabular}{|c|c|c|c|c|}
\hline & \multicolumn{2}{|c|}{ Personal } & \multicolumn{2}{|c|}{ Académico } \\
\hline & $\mathbf{x}$ & Des. St. & $\mathbf{x}$ & Des. St. \\
\hline $\begin{array}{l}\text { Uso Ord. } \\
\text { mesa }\end{array}$ & 2.54 & 1.437 & 2.56 & 1.26 \\
\hline $\begin{array}{l}\text { Uso Ord. } \\
\text { Portátil }\end{array}$ & 4.34 & 1.120 & 4.48 & .74 \\
\hline Uso Tableta & 2.81 & 1.729 & 2.20 & 1.53 \\
\hline $\begin{array}{l}\text { Uso } \\
\text { Smartphone }\end{array}$ & 4.81 & .645 & 2.41 & 1.12 \\
\hline $\begin{array}{l}\text { Uso Smart- } \\
\text { watch }\end{array}$ & 1.28 & 1.054 & 1.09 & .407 \\
\hline $\begin{array}{l}\text { Uso Disp. } \\
\text { Real. Virt. }\end{array}$ & 1.00 & .000 & 1.09 & .407 \\
\hline Uso Disp. 3D & 1.00 & .000 & 1.09 & .407 \\
\hline Uso Juegos & 1.60 & 1.052 & 1.09 & .407 \\
\hline $\begin{array}{l}\text { Uso Disp. } \\
\text { Stream }\end{array}$ & 1.74 & 1.198 & 1.09 & .407 \\
\hline Uso Disp. Voz & 1.00 & .000 & 1.09 & .407 \\
\hline
\end{tabular}

\subsection{Uso de las tecnologías en el ámbito personal y académico}

Tal y como se describe en el apartado anterior, los datos obtenidos indican que los estudiantes disponen de las herramientas y recursos necesarios para utilizar la tecnología tanto en el ámbito académico como social, por lo que las diferencias de uso que se han encontrado no pueden atribuirse a este hecho.

Los resultados señalan que el uso de las tecnologías en el ámbito personal y académico difiere en función del dispositivo (H3). Mientras los estudiantes utilizan de forma indiferente el ordenador portátil o las tablets para el ámbito académico y personal, el uso del Smartphone es diferente para el ámbito privado y académico (tabla 6 ).

Esa diferenciación también se mantiene en función del género, así tanto hombres como mujeres utilizan su Smartphone más para su vida personalsocial (VS) que académica (VA). Por el contrario, no se observan diferencias en el uso del resto de dispositivos, ni para el ámbito académico ni para el ámbi- 
to personal (tabla 7). Solo se observa una ligera diferenciación en cuanto al dispositivo utilizado; hay una ligera preferencia por dispositivos móviles de parte de las mujeres.

\section{DISCUSIÓN Y CONCLUSIONES}

TABLA 7. Media y DT del uso de cada dispositivo en función del contexto y género

\begin{tabular}{|c|c|c|c|c|}
\hline & & $\mathbf{N}$ & Media & $\begin{array}{c}\text { Desv. } \\
\text { Desviación }\end{array}$ \\
\hline \multirow[t]{2}{*}{ Uso Ord. Mesa VS } & $\mathrm{H}$ & 65 & 3.12 & 1.536 \\
\hline & $M$ & 121 & 2.22 & 1.281 \\
\hline \multirow[t]{2}{*}{ Uso Portatil VS } & $\mathrm{H}$ & 65 & 3.95 & 1.328 \\
\hline & M & 121 & 4.55 & .931 \\
\hline \multirow[t]{2}{*}{ Uso Tableta VS } & $\mathrm{H}$ & 65 & 2.43 & 1.686 \\
\hline & M & 121 & 3.01 & 1.725 \\
\hline \multirow[t]{2}{*}{ Uso Smartp VS } & $\mathrm{H}$ & 65 & 4.94 & .242 \\
\hline & $M$ & 121 & 4.74 & .772 \\
\hline \multirow[t]{2}{*}{ Uso OrdMesa VA } & $\mathrm{H}$ & 65 & 3.00 & 1.436 \\
\hline & M & 121 & 2.32 & 1.097 \\
\hline \multirow[t]{2}{*}{ Uso Portatil VA } & $\mathrm{H}$ & 65 & 4.23 & .932 \\
\hline & M & 121 & 4.62 & .581 \\
\hline \multirow[t]{2}{*}{ Uso Tableta VA } & $\mathrm{H}$ & 65 & 2.05 & 1.363 \\
\hline & M & 121 & 2.29 & 1.610 \\
\hline \multirow[t]{2}{*}{ Uso Smartp VA } & $\mathrm{H}$ & 65 & 2.78 & 1.256 \\
\hline & $M$ & 121 & 2.29 & 1.012 \\
\hline
\end{tabular}

Por lo tanto, la resistencia al cambio incide en la aceptación a la tecnología (García-Peñalvo, \& Montoya, 2017; Muñoz et al., 2017; Zempoalteca et al., 2018).

Cabe destacar que el alumnado ha opinado solo en base a aquella tecnología educativa y prácticas que conoce y ha mostrado un desconocimiento importante sobre las posibilidades que dan las diferentes plataformas de la universidad, así como de las prácticas activas que se pueden llevar a través de ellas. De estos datos se concluye que el alumnado sólo conoce las plataformas y aplicaciones que son obligatorias para continuar con sus estudios y su participación en ellas es pasiva. Esto coincide con estudios que ponen de manifiesto la relación que hay entre estilos de aprendizaje y preferencias de enseñanza. Así, en el estudio desarrollado por Baeten et al. (2016) encuentran que aquellos estudiantes que realizan un aprendizaje superficial prefieren una enseñanza dirigida y un estilo pasivo, mientras que aquellos estudiantes que realizan un aprendizaje profundo prefieren la construcción del conocimiento y el aprendizaje cooperativo. Sin embargo, hay que ser cautelosos con esta conclusión puesto que no sabemos qué uso de los medios digitales realiza el profesorado y cómo influye ello en la actitud y uso de la tecnología por parte del alumnado y en su estilo de aprendizaje. De hecho, el estilo de aprendizaje de los estudiantes podría variar de superficial a 
profundo, y viceversa, en función de las demandas del profesorado y de la forma de evaluar (Baeten et al., 2016; Gargallo López et al., 2015; Navaridas, \& Jiménez, 2016), así como de la elección de recursos (Recio et al., 2020). Por lo tanto, en líneas futuras habría que estudiar el estilo de enseñanza del profesorado, qué uso de la tecnología realiza y qué metodología sigue, para analizar en profundidad la respuesta del alumnado.

Por otra parte, al igual que para su vida académica, el alumnado identifica las TIC como un elemento importante de cara a su futura vida laboral, ya que, consideran les ayudará a desarrollarse profesionalmente. Sin embargo, ya se ha visto que el considerar la tecnología importante, no se traduce en utilizarla e integrarla. Eso nos lleva a cuestionar si al igual que en el caso del proceso de aprendizaje, aun siendo conscientes de la importancia de la tecnología para su futuro profesional, preferirán no integrarla en su práctica. Así pues, en líneas futuras habría que analizar si esa actitud influye en el uso futuro de la tecnología, tal y como lo han analizado Prieto et al., (2017).

Por último, se subraya que los resultados de este estudio varían sustancialmente del plano académico al plano personal. En el caso del plano personal, el uso de la tecnología por parte del alumnado para relacionarse y comunicarse socialmente es diario, proactivo y se realiza de igual forma por hombres que por mujeres. Esta diferencia de actitud y uso de las TIC entre el plano personal y el académico concuerda con otras investigaciones en las que se demuestra que los estudiantes no trasladan el uso y actitud favorable respecto al uso de las TIC de la vida personal y principalmente social, a otros ámbitos como el académico o laboral (Corrin, 2014; Kennedy et al., 2007; Waycott et al., 2010).

Uno de los argumentos podría ser que no conocen el alcance de las herramientas disponibles en la Universidad o las potencialidades que ofrecen éstas, ya que, los resultados indican que únicamente conocen dos plataformas propias de la Universidad y que el uso de las mismas está dirigido a gestión y/o repositorio.

Así pues, en líneas futuras habría que analizar por qué los recursos disponibles en la Universidad no llegan hasta el alumnado y cómo influye el uso que hace el profesorado en la actitud del alumnado sobre ellas. Necesitaríamos estudiar si desde la universidad se está dando la visibilidad y formación adecuada al profesorado y alumnado.

Por otra parte, habría que analizar también por qué el profesorado no hace un uso (adecuado) de los recursos tecnológicos disponibles y de cómo integra éstos de forma constructiva en sus asignaturas. La sola introducción de las TIC en la actividad docente no representa un cambio innovador (Bates, 2009), se han de dar cambios también en la metodología, en el sistema de evaluación y en el rol del profesor y de los estudiantes (Romero et al., 2017). Es necesario analizar por qué los estudiantes prefieren una formación mediada sin tecnología cuando en el resto de ámbitos se desenvuelve mejor con ella.

\section{REFERENCIAS}

Baeten, M., Dochy, F., Struyven, K., Parmentier, E., \& Vanderbruggen, A. (2016). Student-centred learning environments: an investigation into student teachers' instructional preferences and approaches to learning. Learning Environments Research, 19(1), 43-62. 10.1007/s10984-015-9190-5
Bates, T. (2009). Promesas y mitos del aprendizaje virtual en la educación post-secundaria. In M. Castells (Ed.), La sociedad Red: una visión global (pp. 335-359). Alianza

Becker, S., Brown, M., Dahlstrom, E., Davis, A., DePaul, K., Diaz, V., \& Pomerantz. J. (2018). NMC Horizon Report: Higher Education Edition. EDUCAUSE, 2018 
Bennett, S., Maton, K., \& Kervin, L. (2008). The ‘digital natives' debate: A critical review of the evidence. British Journal of Educational Technology, 39(5), 775-786. https://doi.org/10.1111/ j.1467-8535.2007.00793.x

Boyd, D. (2014). It's complicate: The social lives of networked teens. Yale University Press.

Brooks, D., \& Pomerantz, J. (2017). ECAR Study of Undergraduate Students and Information Technology. Research report. ECAR.

Corrin, L. (2014). Examining digital natives: an investigation of university students' engagement with technology [Doctoral dissertation, University of Wollongong]. http://ro.uow.edu. au/theses/4121

Erstad, O. (2010). Educating the digital generation. Exploring media literacy for the 21st century. Nordic Journal of Digital Literacy, 10, 85-102.

Escobar-Pérez, J., \& Cuervo-Martínez, A. (2008). Validez de contenido y juicio de expertos: una aproximación a su utilización. Avances en medición, 6(1), 27-36.

Gallardo, E., Bullen, M., \& Marqués, L. (2016). Student Communication and Study Habits of First-Year University Students in the Digital Era. Canadian Journal of Learning and Technology, 42(1), 1-21. https://doi.org/10.21432/t2d047

Gallardo, E. E., Marqués, L., Bullen, M., \& Strijbos, J. W. (2015). Let's talk about digital learners in the digital era. The International Review of research in open and distributed learning, 16(3). https://doi.org/10.19173/irrodl.v16i3.2196

Gisbert, M., \& Esteve, F. (2016). Digital Leaners: la competencia digital de los estudiantes universitarios. La cuestión universitaria, (7), 48-59.

Gross, B., \& Noguera, I. (2015). Fructuoso, Ingrid Noguera. Mirando el futuro: Evolución de las tendencias tecnopedagógicas en Educación Superior. Campus virtuales, 2(2), 130-140.

Helsper, E. J., \& Eynon, R. (2010). Digital natives: Where is the evidence? British Educational Research Journal, 36(3), 503-520. $10.1080 / 01411920902989227$

Hernández, J.P, \& Torrijos, P. (2018). Percepción del profesorado sobre la integración de las Tecnologías de la Información y la Comunicación (TIC) en las modalidades docentes. Influencia del género y la edad. EDMETIC, Revista de Educación Mediática y TIC, 8(1), 128-146. https://doi.org/10.21071/edmetic. v8i1.10537

Hietajärvi, L., Salmela-Aro, K., Tuominen, H., Hakkarainen, K., \& Lonka, K. (2019). Beyond screen time: Multidimensionality of socio-digital participation and relations to academic wellbeing in three educational phases. Computers in Human Behavior, 93, 13-24.https://doi.org/10.1016/j.chb.2018.11.049

Howe, N., \& Strauss, W. (1991). Millennials Rising: The Next Great Generation. Vintage Original.

Kennedy, G., Dalgarno, B., Gray, K., Judd, T., Waycott, J., Bennett, S., \& Chang, R. (2007). The net generation are not big users of web 2.0 technologies: Preliminary findings. En Ascilite (Ed.), ICT: Providing choices for learners and learning. Proceedings ascilite Singapore 2007 (pp. 517-525). Ascilite.

Kumar, S. (2009). Undergraduate Perceptions of the Usefulness of Web 2.0 in Higher Education: Survey Development. En D. Remenyi (Ed.), Proceedings of 8th European Conference on Elearning (pp. 308-314). ECEL

Muñoz, M. L. A., Zamorano, I. V., Riveros, S. M. A., \& Cabero-Almenara, J. (2017). Tipologías para la Innovación tecnológica en Docentes de Educación Superior a partir de un análisis de conglomerados: un estudio exploratorio. Revista de Educación a Distancia, (55), 1-25. https://doi.org/10.6018/red/55/5

Navaridas, F., \& Jiménez, M. A. (2016). Concepciones de los estudiantes sobre la eficacia de los ambientes de aprendizaje universitarios. Revista de Investigación Educativa, 34(2), 503-519. https://doi.org/10.6018/rie.34.2.239481

Pedró, F. (2009). New millennium learners in higher education: evidence and policy implications. Centre for Educational Research and Innovation (CERI). OECD.

Prensky, M. (2001). Digital natives, digital immigrants part 1. On the Horizon, 9(5), 1-6. https://doi. org/10.1108/10748120110424816

Prieto, J. C. S., Migueláñez, S. O., \& García-Peñalvo, F. J. (2017). ¿Utilizarán los futuros docentes las tecnologías móviles? Validación de una propuesta de modelo TAM extendido. Revista de Educación a Distancia, (52), 1-31. https://doi.org/10.6018/red/52/5 
Ramírez-Montoya, M. S., \& García-Peñalvo, F. J. (2017). La integración efectiva del dispositivo móvil en la educación y en el aprendizaje. RIED. Revista Iberoamericana de Educación a Distancia, 20(2), 29-47. https://doi.org/10.5944/ried.20.2.18884

Recio Muñoz, F., Silva Quiroz, J., \& Abricot Marchant, N. (2020). Análisis de la Competencia Digital en la Formación Inicial de estudiantes universitarios: Un estudio de meta-análisis en la Web of Science. Píxel-Bit. Revista De Medios Y Educación, (59), 125-146. https://doi.org/10.12795/pixelbit.77759

Romero, M. R., Castejón, F. J., López, V. M., \& Fraile, A. (2017). Evaluación formativa, competencias comunicativas y TIC en la formación del profesorado. Comunicar, 52(25), 73-82. https:// doi.org/10.3916/c52-2017-07

Romero, A., Tejada, E., \& López, A. (2017). Desarrollo de la competencia TIC a través del Aprendizaje Servicio: una experiencia en la formación del profesorado de educación infantil. Revista Educativa Hekademos, 22, 46-57.

Sánchez, J. J. M., Ruiz, A. B. M., Sánchez, F. A. G., \& Pina, F. H. (2013). Valoración de las TIC por los estudiantes universitarios y su relación con los enfoques de aprendizaje. Revista de investigación educativa, 31(2), 554. https://doi.org/10.6018/ rie.31.2.151891

Tapscott, D. (1998). Growing up digital: the rise of the Net generation. McGraw-Hill.

Tejada, E., \& Maiz, I. (2013). Nuevos escenarios digitales. Las tecnologías de la información y la comunicación aplicadas a la formación y desarrollo curricular. En: J. Barroso \& J. Cabero, (Coords.)., La utilización de las redes sociales desde una perspectiva educativa (pp. 307-319). Pirámide.

Vázquez, S.; Alemán, L., \& Gómez, M. (2016). Uso de las tecnologías de la información y la comunicación en el proceso de enseñanza-aprendizaje. Virtualidad, Educación y Ciencia, 12 (7), 76-84.

Waycott, J., Bennett, S., Kennedy, G., Dalgarno, B., \& Gray, K. (2010). Digital divides? Student and staff perceptions of information and communication technologies. Computers \& Education, 54(4), 1202-1211. https://doi.org/10.1016/j.compedu.2009.11.006
Zempoalteca, B., González, J., Barragán, J., \& Guzmán, T. (2018). Factores que influyen en la incorporación de las Tecnologías de la Información y la Comunicación en universidades públicas: una aproximación desde la autopercepción docente. Revista de la Educación Superior, 47(186), 51-74. https://doi. $\operatorname{org} / 10.36857 /$ resu.2018.186.348 